\title{
Minimalist approach to donor hepatectomy
}

\author{
See Ching Chan $^{1} \cdot$ Sheung Tat Fan ${ }^{1,2}$
}

Received: 14 July 2015/Accepted: 2 September 2015/Published online: 8 October 2015

(C) Asian Pacific Association for the Study of the Liver 2015

In living donor liver transplantation, any donor hepatectomy is a major undertaking, and it is fundamental that the harm brought to the donor is kept to a minimum, while the transplantation is predictably successful. A larger partial liver, which has a relatively good margin of safety, has historically been favored in terms of better recipient recovery, but graft handling at procurement and implantation is also less mature and graft preservation less satisfactory. With the continuous advancement of the specialty, it has become possible to use smaller and smaller grafts in relation to recipient body size, while meeting individual recipient's metabolic demands [1]. As a corollary, the pendulum is swinging back to the use of the left liver [2]; on the other hand, right liver transplantation is feasible for patients who are much larger than their donors.

With respect to donor benefits, when it comes to the amount of liver parenchyma to be removed, the smaller the better, of course. The notion of preserving the donor's gallbladder is in line with this principle. However, it must be evaluated from two perspectives. Does gallbladder preservation compromise graft procurement and thus render the graft suboptimal for transplantation? Will a "retained" gallbladder pose more problems than benefit to the donor in the future?

Sheung Tat Fan

stfan@hku.hk

See Ching Chan

seechingchan@gmail.com

1 Department of Surgery, The University of Hong Kong, 102 Pok Fu Lam Road, Pok Fu Lam, Hong Kong, China

2 Liver Surgery Centre, Hong Kong Sanatorium \& Hospital, 2 Village Road, Happy Valley, Hong Kong, China
During donor right hepatectomy, the line of division of the right hepatic duct is best decided under intraoperative cholangiography guidance. This is to enable inclusion of the most distal portion of the right hepatic duct while keeping a 1-mm ductal stump for closure by careful suturing. If the right hepatic duct is of reasonable length on preoperative biliary magnetic resonance imaging, the latitude of ductal division is assured. If it is rather short, or if it is absent entirely, ductal division without cholangiography guidance can be hazardous to the donor and may render recipient ductal reconstruction difficult and suboptimal. Near-infrared fluorescence cholangiography may be helpful for more precise determination of the line of division of the right hepatic duct [3]. As indocyanine green is excreted into the main ducts, fluorescence makes the ducts more visible, so the surgeon has a better picture of the biliary anatomy. When the division has been completed, the surgeon should gently and carefully probe the liver remnant to verify the integrity of the ducts, including the common hepatic duct and the bifurcation.

The cystic artery most commonly arises from the right hepatic artery. Preservation of the cystic artery may reduce the length obtainable for the right hepatic artery. A gallbladder not hanging freely after removal of the right liver can be indicative of gallbladder torsion, resulting in necrosis. Deprived of innervation, a gallbladder with necrosis or infection may show late presentation of symptoms of discomfort or pain. With the right liver removed, the gallbladder will be surrounded by bowel loops, rendering urgent or elective percutaneous ultrasonography impossible, and imaging by computed tomography or magnetic resonance will be needed. If cholecystitis develops, diagnosis can be delayed.

In donor left hepatectomy, the line of division of the left hepatic duct usually has greater latitude, unless the right 
posterior duct arises from the left duct, which should be identifiable by preoperative magnetic resonance cholangiography. The gallbladder is untouched, remains attached to the gallbladder bed, and is not subject to the risk of torsion. Thus gallbladder preservation is usually accomplished without difficulty [4]. In the experience of an established center, the use of right or left liver grafts made no difference in terms of donor biliary complications, and intraoperative cholangiography by cannulation of the cystic duct was found to help lower the donor biliary complication rate [5]. Donor biliary complications are classified as Grade IIIa or above in the Clavien-Dindo classification of surgical complications, which signifies that they are serious complications [6, 7].

In summary, the minimalist approach to donor hepatectomy is preferred, but can only be applied when the donor liver anatomy favors such an approach. The long-term outcome of gallbladder preservation for a right liver donor is still unknown. Is a gallbladder with suboptimal function more prone to develop gallstones or gallbladder sludge? As noted by Dong et al. [4], these are issues worth looking into prospectively. While in every situation we try to provide the best health care to the altruistic living liver donors, their safety remains the primary concern. Despite the fact that their long-term quality of life after liver donation is likely to be marred by recipient death within 2 years [8], if it so happens, their future should not be compromised by any increased donor risk, short-term or long-term. Dong et al., however, have certainly made a fantastic effort in improving living liver donor outcomes.

\section{References}

1. Chan SC, Lo CM, Ng KK, Fan ST. Alleviating the burden of small-for-size graft in right liver living donor liver transplantation through accumulation of experience. Am J Transplant 2010;10(4):859-867

2. Ishizaki Y, Kawasaki S, Sugo H, Yoshimoto J, Fujiwara N, Imamura H. Left lobe adult-to-adult living donor liver transplantation: should portal inflow modulation be added? Liver Transpl 2012;18(3):305-314

3. Osayi SN, Wendling MR, Drosdeck JM, et al. Near-infrared fluorescent cholangiography facilitates identification of biliary anatomy during laparoscopic cholecystectomy. Surg Endosc 2015;29(2):368-375

4. Dong JH, Ye S, Duan WD, Ji WB, Liang YR. Feasibility of liver graft procurement with donor gallbladder preservation in living donor liver transplantation. Hepatol Int 2015 (epub ahead of print)

5. Iwasaki J, Iida T, Mizumoto M, et al. Donor morbidity in right and left hemiliver living donor liver transplantation: the impact of graft selection and surgical innovation on donor safety. Transpl Int 2014;27(11):1205-1213

6. Dindo D, Demartines N, Clavien PA. Classification of surgical complications: a new proposal with evaluation in a cohort of 6336 patients and results of a survey. Ann Surg 2004;240(2):205-213

7. Chan SC, Chan AC, Sharr WW, et al. Perpetuating proficiency in donor right hepatectomy for living donor liver transplantation. Asian J Surg 2014;37(2):65-72

8. Ladner DP, Dew MA, Forney S, et al. Long-term quality of life after liver donation in the adult to adult living donor liver transplantation cohort study (A2ALL). J Hepatol 2015;62(2):346-353 